\title{
Massive gluon propagator at zero and finite temperature
}

\author{
Attilio Cucchieri $^{a, b}$, David Dudal ${ }^{b}$, Tereza Mendes ${ }^{* a}$ and Nele Vandersickel ${ }^{b}$ \\ ${ }^{a}$ Instituto de Física de São Carlos, Universidade de São Paulo, \\ Caixa Postal 369, 13560-970 São Carlos, SP, Brazil \\ ${ }^{b}$ Ghent University, Department of Physics and Astronomy, \\ Krijgslaan 281-S9, 9000 Gent, Belgium \\ E-mail: attilio@ifsc.usp.br,david.dudal@ugent.be, \\ mendes@ifsc.usp.br, nele.vandersickel@ugent.be
}

\begin{abstract}
We report on our study of the infrared gluon propagator for $\mathrm{SU}(2)$ lattice gauge theory using large lattice volumes. The observed massive behavior is discussed from the point of view of analytic predictions for the zero-temperature case. Such a behavior is still present as the temperature is switched on, but manifests itself differently in the electric and magnetic channels.
\end{abstract}

International Workshop on QCD Green's Functions, Confinement and Phenomenology, September 05-09, 2011

Trento Italy

\footnotetext{
* Speaker.
} 


\section{Introduction}

The massive infrared behavior of the gluon propagator $D\left(p^{2}\right)$ in Landau gauge (see e.g. [1]) has been distinctively observed in lattice simulations using very large volumes a few years ago [2, 3, 4], for SU(2) and SU(3) pure gauge theory (see [5] for a review). This behavior has been recently analyzed in terms of an effective running mass in [6] (see also [7]) and it has been associated to the dimension-two condensates in the so-called refined Gribov-Zwanziger (RGZ) framework (see e.g. [8]) for the $S U(3)$ case in [9]. Here we present the results of our fits to the $S U(2)$ gluon propagator, which can be associated to the RGZ formula. More precisely, we have performed systematic fits to our data in the whole range of available momenta (in the infrared region) using the so-called Gribov-Stingl form $[10,11]$ for modeling the massive behavior of the gluon propagator. This form is a generalization of the Gribov propagator $[12,13]$ - which is based on a pair of purely imaginary complex-conjugate poles - to include pairs of complex-conjugate poles with a nonzero real part, as well as a possible real pole. We have tested several rational forms of this type for $D\left(p^{2}\right)$, and found that a four-parameter expression (in which one of the parameters is a global normalization) gives the best quantitative description of the data in the $4 \mathrm{~d}$ case. In the $3 \mathrm{~d}$ case we need five parameters, one of which again serves as a global normalization. The behavior in the two cases is associated respectively to the simplified and to the general RGZ formulas for $D\left(p^{2}\right)$. In two dimensions, on the contrary, the use of rational forms is not sufficient to describe the data. This case will not be considered here. A more detailed discussion of these zero-temperature fits has been presented recently in [14].

At finite temperature, a similar massive behavior is observed for both the longitudinal (electric) and the transverse (magnetic) gluon propagator in the infrared limit [15, 16, 17, 18, 19, 20, 21, 22]. In this case, we have used a modified Gribov-Stingl form to describe our SU(2) data and to define electric and magnetic screening masses. A recent update on our results can be found in [23]. This study is still preliminary.

In the following, we review the features of the RGZ framework in Section 2, then present our fit results in Section 3. The finite-temperature case is considered in Section 4, followed by our conclusions and the bibliography.

\section{The Refined Gribov-Zwanziger Framework}

The refined Gribov-Zwanziger framework (RGZ) differs from the scenario originally proposed by Gribov [12] and Zwanziger [13] through the introduction of dimension-two condensates [8, 24, $25,26,27]$. In the most general case, four different condensates are considered, i.e.

$$
\left\langle A_{\mu}^{a} A_{\mu}^{a}\right\rangle \rightarrow-m^{2} \quad\left\langle\bar{\varphi}_{i}^{a} \varphi_{i}^{a}\right\rangle \rightarrow M^{2} \quad\left\langle\varphi_{i}^{a} \varphi_{i}^{a}\right\rangle \rightarrow \rho \quad\left\langle\bar{\varphi}_{i}^{a} \bar{\varphi}_{i}^{a}\right\rangle \rightarrow \rho^{\dagger},
$$

where we have listed the dynamical mass associated to each condensate. Note that the condensate $-m^{2}$ is directly related to the gluon condensate $\left\langle g^{2} A^{2}\right\rangle$. In the presence of the four condensates above, the original infrared suppressed gluon propagator in $[12,13]$ is modified as

$$
D\left(p^{2}\right)=\frac{p^{4}+2 M^{2} p^{2}+M^{4}-\left(\rho_{1}^{2}+\rho_{2}^{2}\right)}{p^{6}+p^{4}\left(m^{2}+2 M^{2}\right)+p^{2}\left[2 m^{2} M^{2}+M^{4}+\lambda^{4}-\left(\rho_{1}^{2}+\rho_{2}^{2}\right)\right]+m^{2}\left[M^{4}-\left(\rho_{1}^{2}+\rho_{2}^{2}\right)\right]+\lambda^{4}\left(M^{2}-\rho_{1}\right)} .
$$


where the condensates $m^{2}, M^{2}, \rho$ are described above and $\lambda^{4}$ is related to the Gribov parameter $\gamma$ through $\lambda^{4}=2 g^{2} N_{c} \gamma^{4}$. Also, we have set

$$
\begin{aligned}
\rho & =\rho_{1}+\mathrm{i} \rho_{2} \\
\rho^{\dagger} & =\rho_{1}-\mathrm{i} \rho_{2} .
\end{aligned}
$$

It is interesting to notice that this propagator gets simplified if $\rho=\rho^{\dagger}=\rho_{1}$ (i.e. $\rho_{2}=0$ ), which corresponds to the equality $\langle\overline{\varphi \varphi}\rangle=\langle\varphi \varphi\rangle$ from (2.1). Indeed, in this case one can factorize the quantity $p^{2}+M^{2}-\rho_{1}$ in the numerator and in the denominator of the above formula, obtaining

$$
D\left(p^{2}\right)=\frac{p^{2}+M^{2}+\rho_{1}}{p^{4}+p^{2}\left(M^{2}+m^{2}+\rho_{1}\right)+m^{2}\left(M^{2}+\rho_{1}\right)+\lambda^{4}} .
$$

Note that both Eqs. (2.2) and (2.4) can be decomposed as sums of propagators of the type $\alpha /\left(p^{2}+\omega^{2}\right)$. In particular, we can write Eq. (2.2) as

$$
D\left(p^{2}\right)=\frac{\alpha}{p^{2}+\omega_{1}^{2}}+\frac{\beta}{p^{2}+\omega_{2}^{2}}+\frac{\gamma}{p^{2}+\omega_{3}^{2}} .
$$

To this end, we only need to solve the cubic equation

$$
\begin{aligned}
& x^{3}+x^{2}\left(m^{2}+2 M^{2}\right)+x\left[2 m^{2} M^{2}+M^{4}+\lambda^{4}-\left(\rho_{1}^{2}+\rho_{2}^{2}\right)\right]+m^{2}\left[M^{4}-\left(\rho_{1}^{2}+\rho_{2}^{2}\right)\right] \\
&+\lambda^{4}\left(M^{2}-\rho_{1}\right)=0,
\end{aligned}
$$

obtained by setting $p^{2}=x$ in the denominator of Eq. (2.2), and to find its three roots $\omega_{1}^{2}, \omega_{2}^{2}$ and $\omega_{3}^{2}$. At the same time, the gluon propagator in Eq. (2.4) can be written as

$$
D\left(p^{2}\right)=\frac{\alpha_{+}}{p^{2}+\omega_{+}^{2}}+\frac{\alpha_{-}}{p^{2}+\omega_{-}^{2}},
$$

where we expect to have $\alpha_{-}=\alpha_{+}^{*}$ if $\omega_{-}^{2}=\left(\omega_{+}^{2}\right)^{*}$, i.e. if $\omega_{+}^{2}$ and $\omega_{-}^{2}$ are complex conjugates. Here, $\omega_{ \pm}^{2}$ are the roots of the quadratic equation

$$
x^{2}+x\left(M^{2}+m^{2}+\rho_{1}\right)+m^{2}\left(M^{2}+\rho_{1}\right)+\lambda^{4}=0,
$$

obtained by setting $p^{2}=x$ in the denominator of Eq. (2.4). Clearly, one finds complex-conjugate poles if $\left|M^{2}-m^{2}+\rho_{1}\right|<2 \lambda^{2}$.

Let us remark that rational forms such as (2.2) and (2.4) for the gluon propagator were considered by Stingl [10,11], as a way of accounting for nonperturbative effects in an extended perturbative approach to Euclidean QCD. More precisely, in his treatment, one expresses the proper vertices of the theory as an iterative sequence of functions yielding a self-consistent solution to the Dyson-Schwinger equations. In particular, for the gluon propagator, this sequence is written [see Eq. (2.10) in Ref. [11]] in terms of ratios of polynomials in the variable $p^{2}$, of degree $r$ in the numerator and $r+1$ in the denominator, with $r=0,1,2, \ldots$. This functional form is then related, via operator the product expansion, to the possible existence of vacuum condensates of dimension $2 n$, with $n \geq 1$. At the same time, the associated complex-conjugate poles are interpreted as short-lived elementary excitations of the gluon field $[10,13,11]$. By comparison, in the RGZ framework, one proposes specific forms for the dimension-two condensates - related to the auxiliary fields of the GZ action - and then obtains (at tree level) the rational functions in Eqs. (2.2) and (2.4), which correspond respectively to cases with $r=3$ and 2 in Stingl's iterative sequence. 


\section{Zero-temperature results}

We analyze data for the SU(2) Landau-gauge gluon propagator, produced in 2007 and already discussed in $[3,28,29,5]$, but not systematically fitted until recently. Our run parameters and lattice setup are described in [14]. We note that the lattice spacing $a$ is set by using the $4 \mathrm{~d} \mathrm{SU}(3)$ value for the string tension, as described in [30] and [31] respectively for $d=3$ and 4 . All our runs are in the scaling region. Possible systematic effects due to Gribov copies as well as unquenching effects are not considered here. Finite-volume effects, on the other hand, are well under control and our largest lattice volumes can be already considered as infinite. We notice that, in order to reduce discretization errors due to the breaking of rotational symmetry, we have considered several configurations for the momentum components $p_{\mu}$ and used the improved momentum definition in [32], which does not affect the value of $p^{2}$ in the IR limit, but modifies its value significantly for large momenta. We have checked that the use of improved momenta helps to obtain a better fit to the data in both the $4 \mathrm{~d}$ and the $3 \mathrm{~d}$ cases.

Values of physical parameters (i.e. the condensates and poles introduced in the previous section) are extracted from the data at the largest lattices, with lattice volume $128^{4}$ in $4 \mathrm{~d}$ and $320^{3}$ in $3 \mathrm{~d}$, and lattice spacing respectively of $0.210 \mathrm{fm}$ and $0.268 \mathrm{fm}$. This corresponds to physical volumes of about $(27 \mathrm{fm})^{4}$ and $(85 \mathrm{fm})^{3}$, or equivalently smallest momenta of about $46 \mathrm{MeV}$ and $14 \mathrm{MeV}$, respectively in $4 \mathrm{~d}$ and $3 \mathrm{~d}$.

Our results are summarized below. We refer to [14] for a more complete analysis. We remark that the shown data for $D\left(p^{2}\right)$ are not normalized and that a renormalization condition at a given scale would correspond to a rescaling of the overall factor $C$ in the fitting forms considered below. The condensates and the poles, on the other hand, are not affected by such a renormalization. Also note that, since our largest momentum is of the order of $4 \mathrm{GeV}$, ultraviolet logarithmic corrections are not important to describe the lattice data and they are not included in the fitting functions proposed here. This also avoids the problem of having to regularize the corresponding Landau pole by hand.

In the $4 \mathrm{~d}$ case, our best fit is obtained for a four-parameter fitting function of the simplest Gribov-Stingl form

$$
f_{1}\left(p^{2}\right)=C \frac{p^{2}+s}{p^{4}+u^{2} p^{2}+t^{2}},
$$

which corresponds to the simplified RGZ propagator in Eq. (2.4), modulo the global rescaling factor $C$. The results of the fit for all lattice volumes, using improved momenta, are reported in Table 1. The good quality of the fit is seen by comparing it to the data, as shown (for our largest lattice) in Fig. 1. Let us stress that we are fitting the whole momentum range available and that, for the largest lattice volume, we have 257 data points.

We mention that a test of the more general form of the propagator [given in Eq. (2.2)] considering a six-parameter fitting function leads to an unstable fit, in which most of the parameters are determined with very large errors, suggesting that the function has too many (redundant) parameters. We then reduced the number of parameters by one and introduced the form

$$
f_{4}\left(p^{2}\right)=C \frac{\left(p^{2}+s\right)\left(p^{2}+1\right)}{\left(p^{4}+u^{2} p^{2}+t^{2}\right)\left(p^{2}+k\right)}=C \frac{p^{4}+(s+1) p^{2}+s}{p^{6}+\left(k+u^{2}\right) p^{4}+\left(k u^{2}+t^{2}\right) p^{2}+k t^{2}},
$$




\begin{tabular}{cccccc}
\hline \hline$V$ & $C$ & $u(\mathrm{GeV})$ & $t\left(\mathrm{GeV}^{2}\right)$ & $s\left(\mathrm{GeV}^{2}\right)$ & $\chi^{2} /$ d.o.f. \\
\hline $48^{4}$ & $0.791(0.007)$ & $0.755(0.027)$ & $0.707(0.013)$ & $2.419(0.119)$ & 2.09 \\
\hline $56^{4}$ & $0.801(0.006)$ & $0.734(0.023)$ & $0.696(0.012)$ & $2.305(0.100)$ & 1.92 \\
\hline $64^{4}$ & $0.791(0.007)$ & $0.760(0.024)$ & $0.710(0.012)$ & $2.425(0.108)$ & 2.35 \\
\hline $80^{4}$ & $0.785(0.005)$ & $0.734(0.019)$ & $0.708(0.009)$ & $2.404(0.084)$ & 2.04 \\
\hline $96^{4}$ & $0.795(0.004)$ & $0.717(0.016)$ & $0.694(0.008)$ & $2.291(0.068)$ & 1.66 \\
\hline $128^{4}$ & $0.784(0.005)$ & $0.768(0.017)$ & $0.720(0.009)$ & $2.508(0.078)$ & 1.63 \\
\hline \hline
\end{tabular}

Table 1: Fits of the gluon-propagator data in the $4 \mathrm{~d}$ case, for different lattice volumes, using the fitting function $f_{1}\left(p^{2}\right)$ and improved momenta. We report, besides the value of the fit parameters, the $\chi^{2} /$ d.o.f. obtained in each case. The whole range of momenta was considered for the fit. Errors shown in parentheses correspond to one standard deviation.

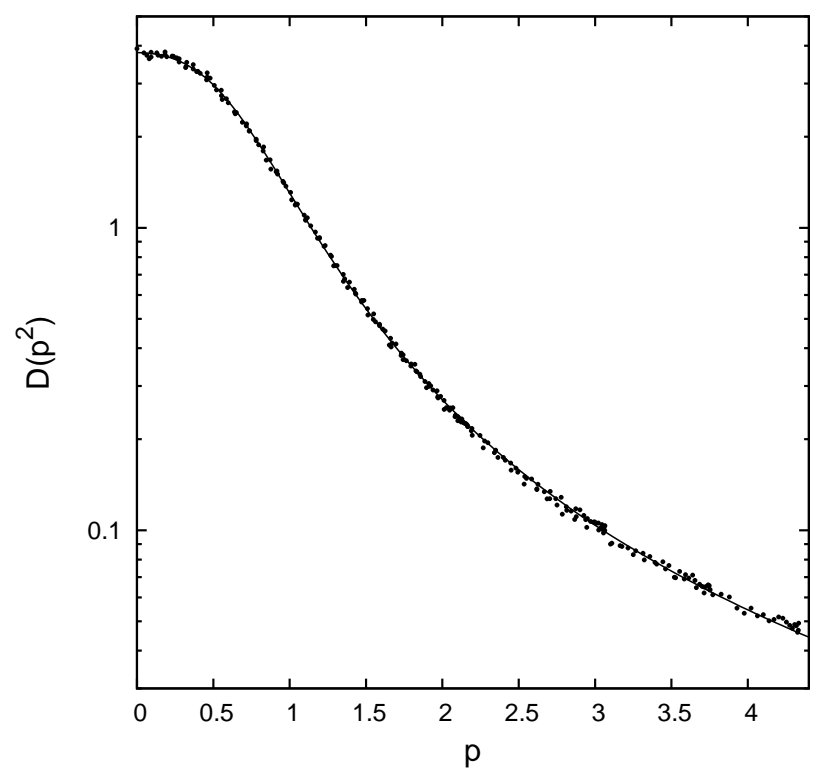

Figure 1: Plot of the $4 \mathrm{~d}$ gluon propagator $D\left(p^{2}\right)$ (in $\mathrm{GeV}^{-2}$ ) as a function of the (improved) momentum $p$ (in $\mathrm{GeV}$ ) for the lattice volume $V=128^{4}$. We also show the fitting function $f_{1}\left(p^{2}\right)$. Note the logarithmic scale on the $y$ axis.

which is written as a simple generalization of $f_{1}\left(p^{2}\right)$ in Eq. (3.1). In this case the fits look reasonable, but the errors are larger and the $\chi^{2} /$ d.o.f. is not better for the five-parameter fit compared to the four-parameter fit, indicating that the latter is more stable. Also, the fits results suggest a very small (and imaginary) value for $\rho_{2}$, implying that $\rho$ is real and thus supporting the simpler form in Eq. (2.4), fitted above using the function $f_{1}\left(p^{2}\right)$.

In order to extract the value of the condensates described in Section 2 above, we thus consider only the fit results for $f_{1}\left(p^{2}\right)$ and the volume $V=128^{4}$ (using improved momenta), reported in the last row of Table 1 and plotted in Fig. 1. By setting $f_{1}\left(p^{2}\right)$ equal to the RGZ propagator in Eq. (2.4) (modulo the global factor $C$ ), we find for the condensates the values reported in Table 2. We 


\begin{tabular}{cccc}
\hline \hline parameter & propagation of error & Monte Carlo analysis & bootstrap analysis \\
\hline$M^{2}+\rho_{1}\left(\mathrm{GeV}^{2}\right)$ & $2.51(8)$ & $2.51(8)$ & $2.3(3)$ \\
\hline$m^{2}\left(\mathrm{GeV}^{2}\right)$ & $-1.92(9)$ & $-1.92(9)$ & $-1.7(2)$ \\
\hline$\lambda^{4}\left(\mathrm{GeV}^{4}\right)$ & $5.3(9)$ & $5.3(4)$ & $4.5(9)$ \\
\hline$a$ & $0.392(3)$ & $0.392(2)$ & $0.38(1)$ \\
\hline$b$ & $1.32(7)$ & $1.32(5)$ & $1.20(7)$ \\
\hline$v\left(\mathrm{GeV}^{2}\right)$ & $0.29(2)$ & $0.29(2)$ & $0.29(3)$ \\
\hline$w\left(\mathrm{GeV}^{2}\right)$ & $0.66(2)$ & $0.66(1)$ & $0.64(2)$ \\
\hline \hline
\end{tabular}

Table 2: Estimates of the parameters of the simplified RGZ gluon propagator in Eq. (2.4) and of the function $f_{2}\left(p^{2}\right)$, obtained from fits to the equivalent form $f_{1}\left(p^{2}\right)$. Errors are calculated using propagation of error, a Monte Carlo analysis and a bootstrap analysis. In all cases we considered the volume $V=128^{4}$ and improved momenta.

see that $\left|M^{2}-m^{2}+\rho_{1}\right|<2 \lambda^{2}$, justifying our expectation that the propagator may be decomposed in terms of a pair of complex-conjugate poles. We can thus write [see Eq. (2.7)]

$$
f_{2}\left(p^{2}\right)=\frac{\alpha_{+}}{p^{2}+\omega_{+}^{2}}+\frac{\alpha_{-}}{p^{2}+\omega_{-}^{2}}=\frac{2 a p^{2}+2(a v+b w)}{p^{4}+2 v p^{2}+v^{2}+w^{2}},
$$

with $\alpha_{ \pm}=a \pm i b$ and $\omega_{ \pm}^{2}=v \pm i w$. The results for the parameters $a, b, v$ and $w$ are also shown in Table 2. We note that the errors (given in parentheses) correspond to one standard deviation and were evaluated in three different ways: by propagation of error, by a Monte Carlo error analysis and by a bootstrap analysis. We refer to [14] for details of these procedures. Clearly, all results obtained agree within errors. We see that the poles are complex conjugates whose imaginary part is more than twice their real part. We recall that a Gribov propagator would have a null real part.

Let us mention that the values obtained here for $M^{2}+\rho_{1}, m^{2}$ and $\lambda^{4}$ are in good quantitative agreement with the corresponding values - respectively indicated with $M^{2}, m^{2}$ and $2 g^{2} N \gamma^{4}-$ reported in Ref. [9] for the SU(3) case. ${ }^{1}$ Also, as remarked above, the condensate $m^{2}$ may be used to obtain a value for the gluon condensate $\left\langle g^{2} A^{2}\right\rangle$, through the relation (see e.g. [9])

$$
\left\langle g^{2} A^{2}\right\rangle=-\frac{9}{13} \frac{N_{c}^{2}-1}{N_{c}} m^{2} .
$$

In our case, the value $m^{2}=-1.92(9)$ from Table 2 (using propagation of error) yields $\left\langle g^{2} A^{2}\right\rangle=$ $1.99(9) \mathrm{GeV}^{2}$.

In the $3 \mathrm{~d}$ case the simplified fitting form $f_{1}\left(p^{2}\right)$ in Eq. (3.1) is not able to describe well the lattice data. Indeed, even using improved momenta, the $\chi^{2} /$ d.o.f. values obtained are quite large. Moreover, as can be seen in Fig. 2, the fit clearly fails in the IR region. ${ }^{2}$ The situation improves by considering the (five-parameter) fitting function $f_{4}\left(p^{2}\right)$ in Eq. (3.2) above, as can be seen from the results reported in Table 3, obtained using improved momenta. Let us mention that we have

\footnotetext{
${ }^{1}$ For comparison with our values in Table 2, the SU(3) condensates from [9] are respectively 2.15(13) $\mathrm{GeV}^{2}$, $-1.81(14) \mathrm{GeV}^{2}$ and $4.16(38) \mathrm{GeV}^{4}$.

${ }^{2}$ In order to highlight the results at small momenta, here and in Fig. 3 we present the plot with a logarithmic scale on both axes.
} 


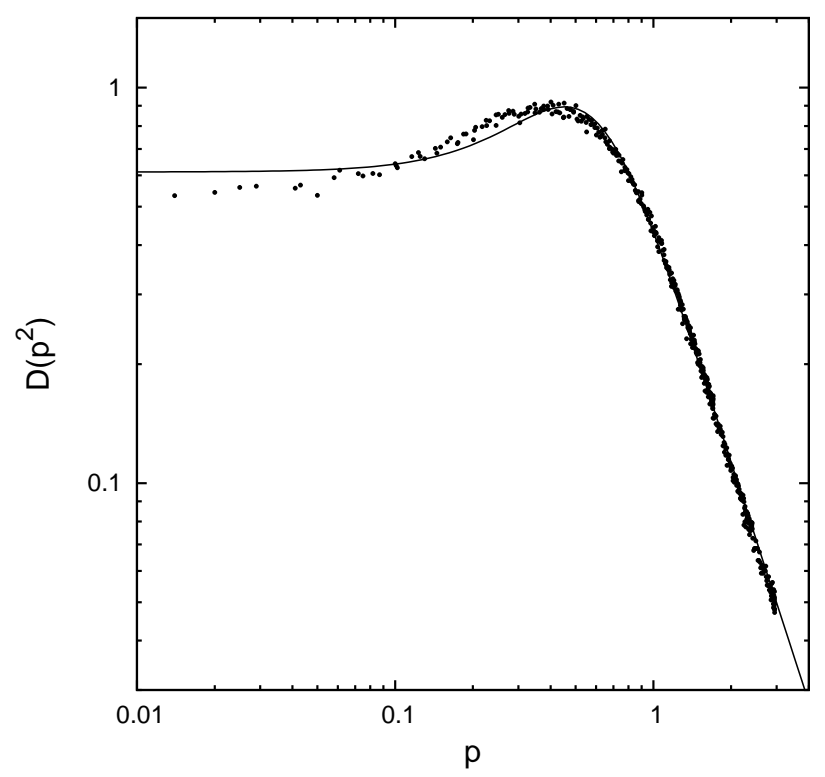

Figure 2: Plot of the $3 \mathrm{~d}$ gluon propagator $D\left(p^{2}\right)$ (in $\mathrm{GeV}^{-1}$ ) as a function of the (improved) momentum $p$ (in $\mathrm{GeV}$ ) for the lattice volume $V=320^{3}$. We also show the fitting function $f_{1}\left(p^{2}\right)$. Note the logarithmic scale on both axes.

\begin{tabular}{cccccc}
\hline \hline$V$ & $C(\mathrm{GeV})$ & $u(\mathrm{GeV})$ & $t\left(\mathrm{GeV}^{2}\right)$ & $s\left(\mathrm{GeV}^{2}\right)$ & $k\left(\mathrm{GeV}^{2}\right)$ \\
\hline $140^{3}$ & $0.407(0.001)$ & $0.654(0.008)$ & $0.623(0.004)$ & $0.022(0.002)$ & $0.041(0.003)$ \\
\hline $200^{3}$ & $0.407(0.001)$ & $0.655(0.007)$ & $0.623(0.004)$ & $0.024(0.002)$ & $0.043(0.003)$ \\
\hline $240^{3}$ & $0.408(0.001)$ & $0.662(0.007)$ & $0.620(0.004)$ & $0.025(0.002)$ & $0.047(0.003)$ \\
\hline $320^{3}$ & $0.408(0.001)$ & $0.656(0.008)$ & $0.619(0.005)$ & $0.023(0.002)$ & $0.046(0.004)$ \\
\hline \hline
\end{tabular}

Table 3: Fits of the gluon-propagator data in the $3 \mathrm{~d}$ case, for different lattice volumes, using the fitting function $f_{4}\left(p^{2}\right)$ and improved momenta. The $\chi^{2} /$ d.o.f. is about 1 for the lattice volume $320^{3}$. The whole range of momenta was considered for the fit. Errors shown in parentheses correspond to one standard deviation.

also tried a six-parameter fit to a more general function, obtained by substituting $\left(p^{2}+1\right)$ in the numerator of $f_{4}\left(p^{2}\right)$ in Eq. (3.2) by $\left(p^{2}+l\right)$. In this case we obtain a good fit (with $\chi^{2} /$ d.o.f. around 1), with $l \approx 1$ and values of the other parameters that are consistent with the ones in Table 3 , indicating that the latter fit is preferable.

In order to evaluate the condensates of the RGZ model, we thus consider only the results from the fit using $f_{4}\left(p^{2}\right)$, given for the lattice size $N=320$ in the last row of Table 3 and plotted in Fig. 3. By setting $f_{4}\left(p^{2}\right)$ [see Eq. 3.2)] equal to the RGZ propagator (2.2) modulo the global factor $C$, we find the values for the condensates in Table 4. Note that, using this fitting form, we are able to evaluate $M^{2}, \rho_{1}$ and $|\rho|$ (and therefore $\rho_{2}$ ) separately. In this case, we can see that $\rho_{2} \neq 0$ and $\rho$ is indeed a complex quantity. This is consistent with the fact that the (four-parameter) fit to the simplified form $f_{1}\left(p^{2}\right)$ fails, as seen above. Finally, we decompose the propagator as in Eq. (2.5) 


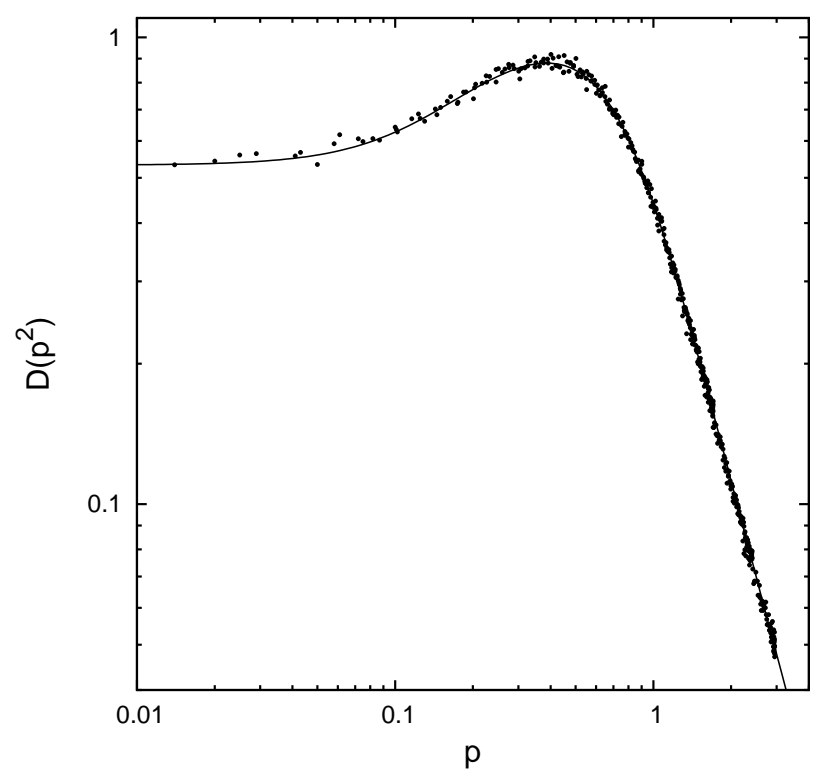

Figure 3: Plot of the $3 \mathrm{~d}$ gluon propagator $D\left(p^{2}\right)$ (in $\mathrm{GeV}^{-1}$ ) as a function of the (improved) momentum $p$ (in $\mathrm{GeV}$ ) for the lattice volume $V=320^{3}$. We also show the fitting function $f_{4}\left(p^{2}\right)$. Note the logarithmic scale on both axes.

with $\beta=a+i b, \gamma=a-i b, \omega_{2}^{2}=v+i w$ e $\omega_{3}^{2}=v-i w$, i.e. we consider the function

$$
f_{6}\left(p^{2}\right)=\frac{\alpha}{p^{2}+\omega_{1}^{2}}+\frac{2 a p^{2}+2(a v+b w)}{p^{4}+2 v p^{2}+v^{2}+w^{2}} .
$$

The corresponding results are also reported ${ }^{3}$ in Table 4 . Also in this case we have performed the error analysis in three different ways: propagation of error, Monte Carlo error analysis and bootstrap analysis (see [14] for details). Note that the imaginary part $w$ of the complex-conjugate poles is more than twice the value of their real part $v$, as in the $4 \mathrm{~d}$ case. Note also that the mass $\omega_{1}$ and the residue $\alpha$ associated with the real pole are very small. Moreover, $\alpha$ is negative, which may be associated with violation of reflection positivity, indicating that this mass cannot correspond to a physical degree of freedom.

\section{Finite temperature}

We have used a modified Gribov-Stingl expression to fit our infrared data for finite-temperature Landau-gauge $\mathrm{SU}(2)$ gluon propagators (in $3+1$ dimensions) reported in [18, 20, 23]. For both the longitudinal (electric) propagator $D_{L}\left(p^{2}\right)$ and for the transverse (magnetic) propagator $D_{T}\left(p^{2}\right)$, we consider the five-parameter fitting form ${ }^{4}$

$$
D_{L, T}\left(p^{2}\right)=C \frac{1+d p^{2 \eta}}{\left(p^{2}+a\right)^{2}+b^{2}} .
$$

\footnotetext{
${ }^{3}$ Clearly, we have $\omega_{1}^{2}=k$ from $f_{4}\left(p^{2}\right)$.

${ }^{4}$ Note that, as in the previous section, the global constant $C$ is fixed (for given values of $a, b, d, \eta$ ) by the renormalization condition, so that there are only four free parameters in (4.1).
} 


\begin{tabular}{cccc}
\hline \hline parameter & propagation of error & Monte Carlo analysis & bootstrap analysis \\
\hline$M^{2}\left(\mathrm{GeV}^{2}\right)$ & $0.512(1)$ & $0.512(1)$ & $0.513(1)$ \\
\hline$m^{2}\left(\mathrm{GeV}^{2}\right)$ & $-0.55(1)$ & $-0.55(1)$ & $-0.52(2)$ \\
\hline$\lambda^{4}\left(\mathrm{GeV}^{4}\right)$ & $0.94(1)$ & $0.94(1)$ & $0.91(3)$ \\
\hline$\rho_{1}\left(\mathrm{GeV}^{2}\right)$ & $0.479(2)$ & $0.479(2)$ & $0.477(2)$ \\
\hline$\rho_{2}\left(\mathrm{GeV}^{2}\right)$ & $0.09(1)$ & $0.094(9)$ & $0.100(6)$ \\
\hline$\alpha\left(\mathrm{GeV}^{2}\right)$ & $-0.024(5)$ & $-0.024(5)$ & $-0.029(4)$ \\
\hline$\omega_{1}^{2}\left(\mathrm{GeV}^{2}\right)$ & $0.046(4)$ & $0.046(4)$ & $0.046(4)$ \\
\hline$a(\mathrm{GeV})$ & $0.216(3)$ & $0.216(2)$ & $0.220(4)$ \\
\hline$b\left(\mathrm{GeV}^{2}\right)$ & $0.27(5)$ & $0.271(3)$ & $0.275(3)$ \\
\hline$v\left(\mathrm{GeV}^{2}\right)$ & $0.215(5)$ & $0.215(5)$ & $0.23(1)$ \\
\hline$w\left(\mathrm{GeV}^{2}\right)$ & $0.580(6)$ & $0.580(6)$ & $0.57(1)$ \\
\hline \hline
\end{tabular}

Table 4: Estimates of the parameters of the general RGZ propagator in Eq. (2.1) and of the function $f_{6}\left(p^{2}\right)$, obtained from fits to the equivalent form $f_{4}\left(p^{2}\right)$. Errors are calculated using propagation of error, a Monte Carlo analysis and a bootstrap analysis. In all cases we considered the volume $V=320^{3}$ and improved momenta.

This form allows for two (complex-conjugate) poles, with masses $m^{2}=a \pm i b$, where $m=$ $m_{R}+i m_{I}$. The mass $m$ thus depends only on $a, b$ and not on the normalization $C$. The parameter $\eta$ should be 1 if the fitting form also describes the large-momenta region (from our infrared data we get $\eta \neq 1$ ). Recall that at high temperatures one usually defines the electric screening mass as the scale determining the exponential decrease of the real-space propagator at large distances, which is equivalent to $D_{L}(0)^{-1 / 2}$ in the case of a real pole. We therefore expect to observe $m_{I} \rightarrow 0$ (i.e. $b \rightarrow 0$ ) for the longitudinal gluon propagator at high temperature. Note that, if the propagator has the above form (with nonzero $b$ ), then the screening mass defined by $D_{L}(0)^{-1 / 2}=\sqrt{\left(a^{2}+b^{2}\right) / C}$ mixes the complex and imaginary masses $m_{R}$ and $m_{I}$ and depends on the (a priori arbitrary) normalization $C$.

We generally find good fits to the modified Gribov-Stingl form above (including the full range of momenta), with nonzero real and imaginary parts of the pole masses in all cases. For the transverse propagator $D_{T}\left(p^{2}\right)$, the masses $m_{R}$ and $m_{I}$ are of comparable size (around 0.6 and $0.4 \mathrm{GeV}$ respectively). The same holds for $D_{L}\left(p^{2}\right)$, but in this case the relative size of the imaginary mass seems to decrease with increasing temperature. A detailed discussion of the associated masses $m_{R}$, $m_{I}$ is postponed to a forthcoming study [33], as we are presently considering variants of the above fitting form inspired by the zero-temperature forms considered in the previous sections.

We show our fits, together with the data, for several values of the temperature $T$ (given in terms of the critical temperature $\left.T_{c}\right)$ in Fig. 4 . We see that $D_{L}\left(p^{2}\right)$ increases as the temperature is switched on, while $D_{T}\left(p^{2}\right)$ decreases slightly, showing a clear turnover point at around $350 \mathrm{MeV}$. It is interesting to notice that the infrared behavior of $D_{L}\left(p^{2}\right)$ remains unchanged (within errors) from $0.5 T_{c}$ to $T_{c}$, as shown in the bottom right plot in the figure. (The curves shown are for lattice parameters $\beta=2.299,2.515$ and lattice volumes $96^{3} \times 8,192^{3} \times 8$ respectively for the temperatures $0.5 T_{c}$ and $1.01 T_{c}$.) In fact, after reducing the severe systematic effects that are observed around 

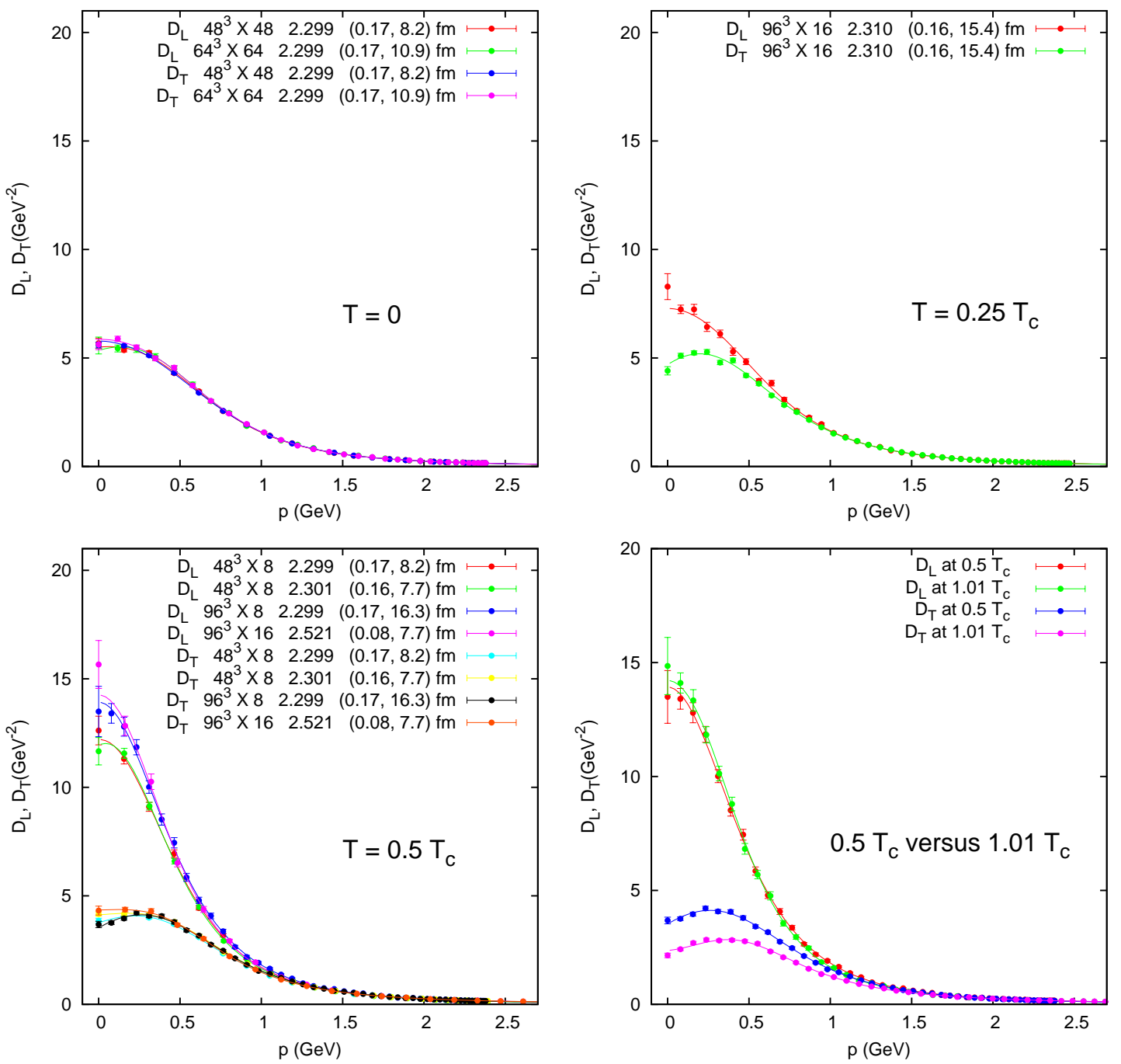

Figure 4: Longitudinal and transverse gluon propagators at $T=0$ (top left), $T=0.25 T_{c}$ (top right) and $T=0.5 T_{c}$ (bottom left). Curves for $T=0.5 T_{c}$ and $1.01 T_{c}$ are shown together for comparison on the bottom right. Values for the lattice volume $N_{s}^{3} \times N_{t}$, the lattice parameter $\beta$, the lattice spacing $a$ and spatial lattice size $L$ (both in fm, in parentheses) are given in the plot labels, with the exception of the bottom right plot, which is described in the text.

$T_{c}$, we find a relatively smooth behavior of $D_{L}\left(p^{2}\right)$ with $T$, which calls into question the sensitivity of the electric propagator to the deconfinement transition.

\section{Conclusions}

By fitting rational functions of $p^{2}$ to the whole range of our (infrared) data for the SU(2) Landau-gauge gluon propagator $D\left(p^{2}\right)$ in four and three space-time dimensions, we are able to obtain estimates for the physical values of the masses in the RGZ framework, as well as to gain a better understanding of the pole structure in the proposed expressions. The data points range from about $4 \mathrm{GeV}$ down to $20-40 \mathrm{MeV}$, which are the smallest simulated momenta to date. In each 
case, we look for the best fit to the data, with the smallest number of independent parameters, and relate them to the condensates in the proposed analytic forms only at the end. Put differently, the predicted dependence of the fit parameters on the condensates is not imposed in the fitting form, but is obtained as a result of the fit.

We find that the $4 \mathrm{~d}$ results are well described by the simplified version of the RGZ gluon propagator in Eq. (2.4), equivalent to the simplest Gribov-Stingl form. This corresponds to a pair of complex-conjugate poles, as opposed to the Gribov propagator, in which the poles would be purely imaginary. The values for the condensates $M^{2}+\rho_{1}, m^{2}$ and $\lambda^{4}$ are in agreement with the ones obtained for the SU(3) case in Ref. [9]. The quantitative agreement between the infrared limit of SU(2) and SU(3) theories was observed numerically before in [4, 34].

In 3d, our fits support the more general form of the RGZ propagator in Eq. (2.2). In this case, the condensate $\rho$ is a complex quantity and there are significant differences in the values of the other condensates and of $\lambda^{4}$ compared to the $4 \mathrm{~d}$ case. Also, in $3 \mathrm{~d}$ one has a real pole mass in addition to the pair of complex-conjugate poles. It is interesting to note that the masses from the complex poles assume similar values in $3 \mathrm{~d}$ and $4 \mathrm{~d}$, with an imaginary part that is more than twice their real part. (We recall that a Gribov propagator would have a null real part.) Note also that the mass and the coefficient associated with the real pole in $3 \mathrm{~d}$ are very small.

Our analysis strongly suggests a pole structure with complex-conjugate masses (with comparable real and imaginary parts) for the infrared gluon propagator in Landau gauge, for zerotemperature (in $4 \mathrm{~d}$ and $3 \mathrm{~d}$ ) and for nonzero temperatures below and around the critical temperature $T_{c}$. As stressed at the end of Section 2, one can interpret this result as describing an unstable particle. In particular (see [14]), in the zero-temperature $4 \mathrm{~d}$ case we obtain the values $m_{g} \approx 550 \mathrm{MeV}$ and $\Gamma_{g} \approx 1180 \mathrm{MeV}$ respectively for the gluon mass and for its width. The very large value for the width $\Gamma_{g}$ may be associated to a lifetime $\tau_{g}$ smaller than $10^{-24} \mathrm{~s}$, supporting the existence of very short-lived excitations of the gluon field.

\section{Acknowledgments}

D. Dudal and N. Vandersickel are supported by the Research-Foundation Flanders (FWO). A. Cucchieri and T. Mendes thank CNPq and FAPESP for partial support. A. Cucchieri also acknowledges financial support from the Special Research Fund of Ghent University (BOF UGent).

\section{References}

[1] A. C. Aguilar, D. Binosi and J. Papavassiliou, Phys. Rev. D 78, 025010 (2008).

[2] I. L. Bogolubsky, E. M. Ilgenfritz, M. Muller-Preussker, A. Sternbeck, PoS LAT2007, 290 (2007).

[3] A. Cucchieri, T. Mendes, PoS LAT2007, 297 (2007).

[4] A. Sternbeck, L. von Smekal, D. B. Leinweber, A. G. Williams, PoS LAT2007, 340 (2007).

[5] A. Cucchieri and T. Mendes, PoS QCD-TNT09, 026 (2009).

[6] A. C. Aguilar, D. Binosi and J. Papavassiliou, JHEP 1201, 050 (2012).

[7] O. Oliveira, P. Bicudo, J. Phys. G38, 045003 (2011). 
[8] D. Dudal, J. A. Gracey, S. P. Sorella, N. Vandersickel, H. Verschelde, Phys. Rev. D78, 065047 (2008).

[9] D. Dudal, O. Oliveira, N. Vandersickel, Phys. Rev. D81, 074505 (2010).

[10] M. Stingl, Phys. Rev. D34, 3863 (1986) [Erratum-ibid. D 36, 651 (1987)].

[11] M. Stingl, Z. Phys. A353, 423 (1996).

[12] V. N. Gribov, Nucl. Phys. B139, 1 (1978).

[13] D. Zwanziger, Nucl. Phys. B364, 127 (1991).

[14] A. Cucchieri, D. Dudal, T. Mendes and N. Vandersickel, arXiv:1111.2327 [hep-lat].

[15] A. Cucchieri, A. Maas and T. Mendes, Phys. Rev. D 75, 076003 (2007).

[16] C. S. Fischer, A. Maas and J. A. Muller, Eur. Phys. J. C 68, 165 (2010).

[17] V. G. Bornyakov and V. K. Mitrjushkin, Phys. Rev. D 84, 094503 (2011).

[18] A. Cucchieri, T. Mendes, PoS LATTICE 2010, 280 (2010).

[19] V. G. Bornyakov and V. K. Mitrjushkin, arXiv:1103.0442 [hep-lat].

[20] A. Cucchieri and T. Mendes, PoS FACESQCD , 007 (2010).

[21] R. Aouane, V. Bornyakov, E. -M. Ilgenfritz, V. Mitrjushkin, M. Muller-Preussker and A. Sternbeck, arXiv:1108.1735 [hep-lat].

[22] A. Maas, J. M. Pawlowski, L. von Smekal and D. Spielmann, arXiv:1110.6340 [hep-lat].

[23] A. Cucchieri, T. Mendes, arXiv:1201.6086 [hep-lat].

[24] D. Dudal, J. A. Gracey, S. P. Sorella, N. Vandersickel, H. Verschelde, Phys. Rev. D78, 125012 (2008).

[25] N. Vandersickel, D. Dudal, S. P. Sorella, PoS FACESQCD, 044 (2010).

[26] N. Vandersickel, [arXiv:1104.1315 [hep-th]].

[27] D. Dudal, S. P. Sorella, N. Vandersickel, Phys. Rev. D84, 065039 (2011).

[28] A. Cucchieri, T. Mendes, Phys. Rev. Lett. 100, 241601 (2008).

[29] A. Cucchieri, T. Mendes, Phys. Rev. D78, 094503 (2008).

[30] A. Cucchieri, T. Mendes, A. R. Taurines, Phys. Rev. D67, 091502 (2003).

[31] J. C. R. Bloch, A. Cucchieri, K. Langfeld, T. Mendes, Nucl. Phys. B687, 76 (2004).

[32] J. P. Ma, Mod. Phys. Lett. A15, 229 (2000).

[33] A. Cucchieri, T. Mendes, in preparation.

[34] A. Cucchieri, T. Mendes, O. Oliveira, P. J. Silva, Phys. Rev. D76, 114507 (2007). 